\title{
Lateral Opening-wedge Distal Femoral Osteotomy: Pain Relief, Functional Improvement, and Survivorship at 5 Years
}

\author{
James I. Cameron MD, Julie C. McCauley MPHc, \\ Arash Y. Kermanshahi MD, William D. Bugbee MD
}

Received: 10 August 2014/Accepted: 9 December 2014/Published online: 24 December 2014

(C) The Association of Bone and Joint Surgeons (B) 2014

\begin{abstract}
Background Distal femoral varus osteotomy may be used to treat valgus knee malalignment or to protect a knee compartment in which cartilage restoration surgery (such as osteochondral or meniscus allografting) has been performed. Medial closing-wedge osteotomy has demonstrated good success in treatment of osteoarthritis in published series, but few studies have evaluated distal femoral lateral opening-wedge osteotomy in terms of correction of deformity, pain and function, and survivorship.
\end{abstract}

Each author certifies that he or she, or a member of his or her immediate family, has no funding or commercial associations (eg, consultancies, stock ownership, equity interest, patent/licensing arrangements, etc) that might pose a conflict of interest in connection with the submitted article.

All ICMJE Conflict of Interest Forms for authors and Clinical Orthopaedics and Related Research ${ }^{\circledR}$ editors and board members are on file with the publication and can be viewed on request.

Clinical Orthopaedics and Related Research ${ }^{\circledR}$ neither advocates nor endorses the use of any treatment, drug, or device. Readers are encouraged to always seek additional information, including FDA-approval status, of any drug or device prior to clinical use. Each author certifies that his or her institution approved the human protocol for this investigation, that all investigations were conducted in conformity with ethical principles of research, and that informed consent for participation in the study was obtained.

This work was performed at Scripps Clinic, La Jolla, CA, USA.

J. I. Cameron, W. D. Bugbee $(\bowtie)$

Division of Orthopaedic Surgery, Scripps Clinic, 10666 North

Torrey Pines Road, MS116, La Jolla, CA 92037, USA

e-mail: bugbee.william@scrippshealth.org

J. C. McCauley

Shiley Center for Orthopaedic Research \& Education at Scripps

Clinic, La Jolla, CA, USA

A. Y. Kermanshahi

Kaiser Permanente, San Diego, CA, USA
Questions/purposes (1) Does lateral opening-wedge osteotomy lead to accurate correction? (2) What pain and function levels do patients experience after lateral openingwedge osteotomy? (3) What are the nonunion, complication, and reoperation rates after lateral opening-wedge osteotomy?

Methods Between 2000 and 2010, we performed 40 distal femoral osteotomies. Two knees (two patients) underwent a medial closing-wedge osteotomy and were excluded from the present study. Thirty-eight knees (97\%) in 36 patients were lateral opening-wedge varus-producing osteotomies; of those, 31 knees $(82 \%)$ in 30 patients had followup at a minimum of 2 years (mean, 5 years; SD, 2; range, $2-12$ years) and comprised the study population. The indications for osteotomy included symptomatic lateral compartment arthritis with clinical valgus deformity or a cartilage or meniscal defect in the lateral compartment with clinical valgus alignment. The study population was stratified into two groups based on reason for osteotomy: patients with isolated symptomatic lateral compartment arthritis (arthritis group; 19 knees [61\%]) and patients who underwent joint preservation procedures including osteochondral allograft transplantation or meniscal allograft transplantation (joint preservation group; 12 knees [39\%]). Data collection from our institution's osteotomy database included patient demographics, lower extremity coronal alignment, and operative details. Pain and function were measured preoperatively and postoperatively using the International Knee Documentation Committee (IKDC) score. Time to radiographic union, complications, and reoperations were recorded.

Results Twenty-one of 31 knees had postoperative radiographic data available for review. Of these, seven of 15 knees in the arthritis group and three of six knees in the joint preservation group were within the correction goal of $\pm 3^{\circ}$ from neutral mechanical alignment. In the arthritis 
group, the mean IKDC total score improved from 47 (SD, 15) preoperatively to $67(\mathrm{SD}, 10)$ postoperatively. In the joint preservation group, the mean IKDC total score improved from $36(\mathrm{SD}, 12)$ preoperatively to $62(\mathrm{SD}, 18)$ postoperatively. One nonunion occurred in the arthritis group. No postoperative complications were experienced. Ten knees in the arthritis group and six knees in the joint preservation group had additional surgery after the osteotomy, consisting primarily of hardware removal, arthroscopy for cartilage-related conditions, or conversion to arthroplasty. Survivorship at 5 years, with conversion to arthroplasty as the endpoint, was $74 \%$ in the arthritis group and $92 \%$ in the joint preservation group.

Conclusions Lateral opening-wedge distal femoral osteotomy was less accurate in correction of valgus deformity than expected, but the procedure was associated with improved knee pain and function scores. Our clinical and radiographic results are comparable to published series evaluating medial closing-wedge distal femoral osteotomy. Achieving our desired correction of $\pm 3^{\circ}$ from neutral alignment was clinically difficult. An improved method of preoperative templating and refinement of the intraoperative technique may improve this. Future studies with more patients and longer followup will provide clarity on this topic.

Level of Evidence Level IV, therapeutic study.

\section{Introduction}

Lower extremity malalignment in association with arthritis or cartilage deficiency is a clinical challenge. Most studies for osteotomies around the knee report on the use of proximal tibial valgus osteotomy for varus deformities [5, 8]. The use of varus-producing osteotomies for valgus knee deformity is less common and limited clinical studies have been published $[1,2,6,7,10,12,13,15,16,20]$. These studies report the correction of deformity and the pain and function of small cohorts of patients undergoing a medial closing-wedge distal femoral osteotomy for treatment of lateral compartment arthritis. Improvement in pain and function of this procedure at intermediate-term followup has been acceptable $[1,2,6,7,12,13,20]$. Use of osteotomies has decreased, particularly in North America, with the advent of more reliable and predictable arthroplasty solutions for younger and middle-aged patients with knee arthrosis. However, with renewed interest in biologic restoration and the use of cartilage restoration techniques, osteotomies have seen an increase in popularity, particularly in younger (age 25-40 years) patients.

The use of an opening-wedge osteotomy on the tibial side for varus deformity has become well established as the favored alternative to the previously more common closingwedge techniques [8]. However, few studies have addressed the analogous opening-wedge technique for femoral osteotomy used to correct valgus deformity $[3,4,11,15,18,19]$. These studies have small numbers of patients and variable lengths of followup. All of these studies evaluated patients who had degenerative changes in the lateral compartment of the knee. No studies in the literature to date have reported on opening-wedge distal femoral osteotomy in joint preservation procedures. The theoretical advantages of the opening-wedge technique over the medial closing-wedge technique include a single bone cut, avoidance of vascular structures, better control of the amount of correction, and more anatomic correction of the typical pathoanatomy of excessive distal femoral valgus [9]. Relative disadvantages include potential for delayed union or nonunion and irritation of the sensitive lateral knee structures by hardware or surgical trauma.

The purpose of our study was to report on a series of opening-wedge distal femoral varus osteotomies used to treat osteoarthritis of the lateral compartment or as an adjunct to correct malalignment with cartilage or meniscal restoration. Specifically, we sought to determine the following: (1) Does lateral opening-wedge osteotomy lead to accurate correction? (2) What pain and function levels do patients experience after lateral opening-wedge osteotomy? (3) What are the nonunion, complication, and reoperation rates after lateral opening-wedge osteotomy?

\section{Materials and Methods}

Our institutional review board-approved osteotomy database was used to identify a case series of 40 knees in 38 patients undergoing distal femoral osteotomy from January 2000 to August 2010. Two knees (two patients) underwent a medial closing-wedge osteotomy and were not included in the present study. Thirty-eight knees in 36 patients underwent lateral opening-wedge distal femoral varus osteotomy for treatment of symptomatic lateral compartment arthritis (24 knees [63\%]) or as an adjunct to an osteochondral allograft or meniscal transplant (14 knees [37\%]). Seven knees in six patients were lost to followup before 2 years and were excluded. The remaining 31 knees (82\%) in 30 patients comprised the study population. The indications for osteotomy included symptomatic lateral compartment arthritis with valgus deformity or an isolated cartilage defect in the lateral compartment with valgus or minimal varus alignment. The study population was stratified into two groups based on reason for osteotomy: patients with isolated symptomatic lateral compartment arthritis (arthritis group; 19 knees [61\%]) and patients who underwent joint preservation procedures including osteochondral allograft transplantation or meniscal allograft transplantation (joint preservation group; 12 knees [39\%]) (Table 1). 
Table 1. Characteristics of the arthritis and the joint preservation groups

\begin{tabular}{lll}
\hline Patient characteristics & $\begin{array}{l}\text { Arthritis group } \\
(\mathrm{N}=19) \\
\text { Mean }(\mathrm{SD}) \\
\text { or number }\end{array}$ & $\begin{array}{l}\text { Joint preservation } \\
\text { group }(\mathrm{N}=12) \\
\text { Mean }(\mathrm{SD}) \\
\text { or number }\end{array}$ \\
\hline Age (years) & $41(9)$ & $26(8)$ \\
Male/female & $7 / 12$ & $4 / 8$ \\
Body mass index $\left(\mathrm{kg} / \mathrm{m}^{2}\right)$ & $25(4)$ & $27(6)$ \\
Diagnosis & & \\
Postmeniscectomy & 8 & 0 \\
Traumatic cartilage injury & 1 & 6 \\
Osteoarthritis & 5 & 1 \\
Tibial plateau fracture & 3 & 1 \\
Osteochondritis dissecans & 0 & 2 \\
Congenital deformity & 1 & 0 \\
Avascular necrosis & 0 & 1 \\
Failed osteochondral allograft & 1 & 0 \\
Failed autologous & 0 & 1 \\
chondrocyte implantation & & 10 \\
Previous surgery on affected & 17 & $3(1)$ \\
knee & & \\
Number of previous surgeries & $3(3)$ & \\
\hline
\end{tabular}

Patients who had any symptoms in the medial or patellofemoral compartment in addition to the lateral compartment were not considered for osteotomy. These patients were either treated nonoperatively or were considered for TKA. Patients with a cartilage defect in the lateral compartment who also had medial knee pain were also not deemed candidates for the osteotomy.

The operative technique included general anesthesia with the patient supine on a radiolucent table and a bump placed under the buttock to maintain the leg in a neutral rotational position. The entire limb, including the iliac crest, was prepped and draped free. A sterile tourniquet was used. An 8to $10-\mathrm{cm}$ incision was made on the lateral distal femur from the lateral epicondyle proximally. The iliotibial band was incised and the vastus lateralis was elevated and dissected off the lateral intermuscular septum to expose the femoral shaft. Under fluoroscopic control, the starting point for the osteotomy was located approximately $3 \mathrm{~cm}$ above the lateral femoral epicondyle and a guide pin was angled medially and distally toward the base of the metaphyseal flare of the medial femoral condyle just above the level of the medial epicondyle. Care was taken to maintain the line above the articular surface of the trochlea. After fluoroscopic confirmation of correct guide pin placement, an osteotomy was performed using an oscillating saw and sharp osteotomies, taking care to maintain approximately $1 \mathrm{~cm}$ of medial bone bridge for osteotomy stability. Once the osteotomy was mobile, an opening-wedge device was placed. The correction

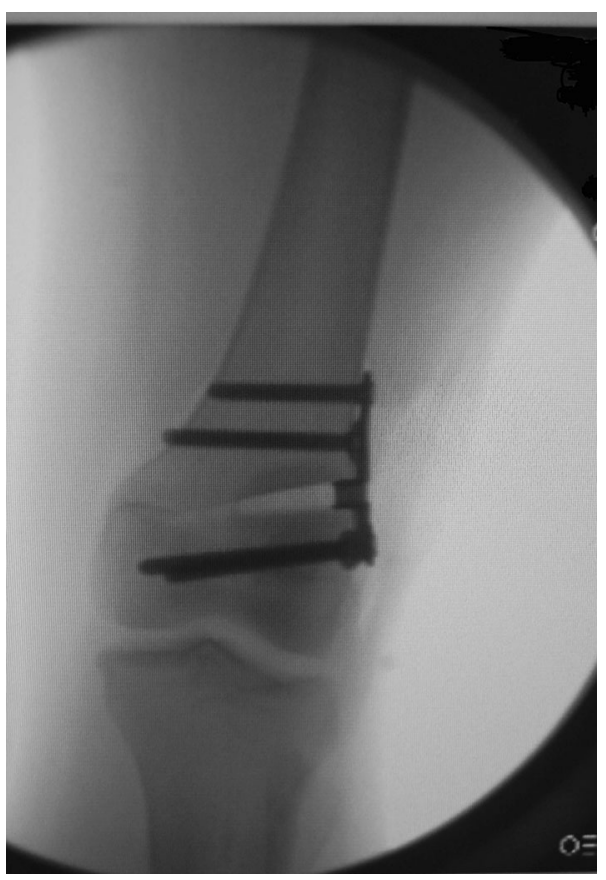

Fig. 1 A fluoroscopic image of an osteotomy is shown after openingwedge and plate and screw fixation.

was slowly created. When the amount of planned correction was obtained at the osteotomy site, lateral fluoroscopic images were obtained to ensure there was no flexion or extension of the osteotomy. Limb alignment was checked fluoroscopically and clinically. The opening-wedge plate was then placed and fixed with four screws (Fig. 1). Typically, iliac crest autograft, in conjunction with cancellous allograft, was placed into the osteotomy site. Routine closure was then performed and the patient was placed into a ROM brace.

Implants used for the osteotomy fixation included 22 Dynafix ${ }^{\circledR}$ VS plates (Biomet, Warsaw, IN, USA), six Puddu plates (Arthrex, Naples, FL, USA), and one TOMOFIX ${ }^{\circledR}$ plate (Synthes, West Chester, PA, USA) (Table 2). Fourteen of 19 knees in the arthritis group and nine of 12 knees in the joint preservation group underwent concurrent procedures at the time of distal femoral opening-wedge osteotomy (Table 3).

Postoperative management included touchdown weightbearing for 6 weeks with no limits to ROM followed by 4 to 6 weeks of progressive weightbearing with the use of crutches. Full weightbearing was allowed at radiographic evidence of healing, typically between 8 and 16 weeks (Fig. 2).

Preoperatively, all patients underwent complete radiographic evaluation including full-length, standing AP radiographs of bilateral lower extremities (some radiographs were done at outside institutions and were not available for alignment measurements for this study). Preoperative templating was performed by one of the authors (WDB) to 
Table 2. Fixation type and graft material

\begin{tabular}{lll}
\hline Fixation type or graft material & $\begin{array}{l}\text { Arthritis group } \\
(\mathrm{N}=19)\end{array}$ & $\begin{array}{l}\text { Joint preservation } \\
\text { group }(\mathrm{N}=12)\end{array}$ \\
\hline Fixation type* $^{*}{ }^{\circledR}$ & 15 & 8 \\
DynaFix $^{\circledR}$ & 3 & 3 \\
Arthrex $^{\circledR}$ & - & 1 \\
TOMOFIX $^{\circledR}$ & 1 & - \\
Other & & \\
Graft material & 5 & 2 \\
Iliac crest autograft alone & 3 & 2 \\
Allograft alone & 11 & 8 \\
Both autograft and allograft &
\end{tabular}

* DynaFix ${ }^{\circledR}$ VS Osteotomy System, EBI, LP, Parsippany, NJ, USA; Arthrex ${ }^{\circledR}$ Osteotomy System, Arthrex Inc, Naples, FL, USA; TOMOFIX ${ }^{\circledR}$ Osteotomy System, Synthes, West Chester, PA, USA.

Table 3. Additional procedures at the time of lateral opening-wedge distal femoral osteotomy

\begin{tabular}{lll}
\hline Additional procedure & $\begin{array}{l}\text { Arthritis group } \\
(\mathrm{N}=19)\end{array}$ & $\begin{array}{l}\text { Joint preservation } \\
\text { group }(\mathrm{N}=12)\end{array}$ \\
\hline $\begin{array}{l}\text { Had additional procedure } \\
\text { Procedure type }\end{array}$ & 14 & 9 \\
$\begin{array}{l}\text { Diagnostic arthroscopy } \\
\text { Osteochondral allograft }\end{array}$ & 8 & 2 \\
Meniscal allograft & 1 & 5 \\
Chondroplasty & - & 1 \\
Meniscectomy & 3 & 1 \\
$\begin{array}{l}\text { Partial meniscectomy and } \\
\text { anterior cruciate ligament }\end{array}$ & 1 & - \\
repair & & - \\
\hline
\end{tabular}

determine the mechanical axis and anatomic axis of the affected lower extremity. The surgical goal was to restore the mechanical alignment to neutral with the mechanical axis through the center of the knee. We used the method of Paley [14] to determine the mechanical axis deviation and amount of required correction. Preoperatively, the amount of correction was estimated using a simplified calculation of $1 \mathrm{~mm}$ of linear correction at the osteotomy site to $1^{\circ}$ of correction of axial alignment. This estimate was adjusted intraoperatively based on both clinical and radiographic analysis. Routine radiographs of the osteotomy site were obtained at followups as well as postoperative long-limb alignment radiographs when possible. Of the 31 knees, 20 (14 in the arthritis group and six in the joint preservation group) had preoperative mechanical axis measurements and 21 (15 in the arthritis group and six in the joint preservation group) had postoperative mechanical axis measurements. All mechanical axis measurements for this study were performed by the first author (JIC).

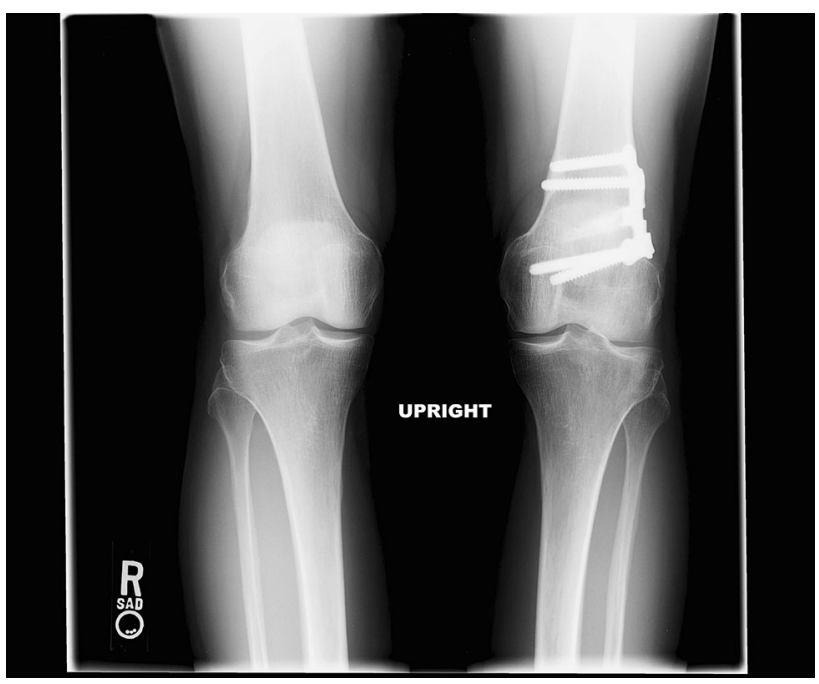

Fig. 2 This image shows radiographic appearance of a healed opening-wedge distal femoral osteotomy.

Patient preoperative and postoperative pain and function were assessed using the International Knee Documentation Committee (IKDC) score. Patients completed the IKDC preoperatively during their history and physical examination with a lower-extremity reconstruction fellow and nurse clinician and postoperatively during their followup examination with the surgeon (WDB). Time to radiographic union, complications, and reoperations were captured. Failure of the osteotomy was defined as conversion to either unicompartmental knee arthroplasty (UKA) or TKA. The two groups of patients (arthritis group and joint preservation group) were considered separately when analyzing the data.

SPSS Version 13.0 (IBM Corporation, Armonk, NY, USA) was used for all statistical analyses. Means and frequencies were calculated to describe patient characteristics of the study population, type of fixation and graft material, amount of intraoperative correction, and mechanical axis alignment. Means and SDs were calculated to describe IKDC pain, function, and total scores preoperatively and at latest followup. However, as a result of the small sample size, it was not appropriate to test the change from preoperatively to followup statistically; thus, no $p$ value is given. Survivorship of the osteotomy, with conversion to arthroplasty (UKA or TKA) as the endpoint, was calculated using the Kaplan-Meier method.

In the arthritis group, the mean followup was 4 years (SD, 3 years; range, 2-12 years). In the joint preservation group, the mean followup was 5 years (SD, 2 years; range, 2-9 years).

\section{Results}

In the arthritis group, the average preoperative mechanical axis was $7^{\circ}$ valgus ( $\mathrm{SD}, 4^{\circ}$; range, $17^{\circ}$ valgus to $1^{\circ}$ varus). 
In the joint preservation group, the average preoperative mechanical axis was $5^{\circ}$ valgus $\left(\mathrm{SD}, 2^{\circ}\right.$; range, $3^{\circ}-8^{\circ}$ valgus). The mean intraoperative correction was $10 \mathrm{~mm}$ (SD, $2 \mathrm{~mm}$ ) for the arthritis group and $9 \mathrm{~mm}(\mathrm{SD}, 3 \mathrm{~mm}$ ) for the joint preservation group. The mean postoperative mechanical axis was $2^{\circ}$ varus $\left(\mathrm{SD}, 4^{\circ}\right.$; range $5^{\circ}$ valgus to $7^{\circ}$ varus) for the arthritis group and $2^{\circ}$ varus $\left(\mathrm{SD}, 4^{\circ}\right.$; range $4^{\circ}$ valgus to $6^{\circ}$ varus) for the joint preservation group. Postoperatively, seven of 15 knees in the arthritis group and three of six knees in the joint preservation group were within the correction goal of $\pm 3^{\circ}$ from neutral mechanical alignment.

Improvements in the IKDC scores were noted postoperatively. In the arthritis group, the mean IKDC pain score improved from $6(\mathrm{SD}, 2)$ to $3(\mathrm{SD}, 3)$, the mean IKDC function score improved from $4(\mathrm{SD}, 1)$ to 7 (SD, 2), and the mean total IKDC score improved from $47(\mathrm{SD}, 15)$ to 67 (SD, 10). In the joint preservation group, the mean IKDC pain score improved from $6(\mathrm{SD}, 1)$ to $2(\mathrm{SD}, 2)$, the mean IKDC function score improved from 3 (SD, 3 ) to 6 (SD, 2), and the mean total IKDC score improved from 36 $(\mathrm{SD}, 12)$ to $62(\mathrm{SD}, 18)$.

One nonunion occurred in the arthritis group (3\%) and was treated with refixation and grafting (Figs. 3, 4) and was ultimately converted to a TKA. All other osteotomies demonstrated radiographic healing by 6 months. No postoperative infections, nerve palsies, or wound complications occurred. No patients noted a leg-length inequality and no persistent symptoms from the iliac crest bone graft site were noted. Ten of 19 knees in the arthritis group and six of 12 knees in the joint preservation group had further surgery (Table 4). Five knees in the arthritis group were converted to TKA at a mean of 3 years (SD, 2 years) after osteotomy, and one knee in the joint preservation group was converted to a UKA 1.7 years after osteotomy. Five-year survivorship was $74 \%$ in the arthritis group and $92 \%$ in the joint preservation group with conversion to arthroplasty as the endpoint.

\section{Discussion}

Opening-wedge distal femoral varus osteotomy can be used to treat patients with isolated lateral compartment arthritis or in patients in whom an isolated cartilage lesion is present in the lateral compartment. Medial opening-wedge proximal tibial osteotomy for varus knee deformity is commonly performed but lateral opening-wedge distal femoral osteotomy for a valgus knee deformity is less common. In this study we report on a cohort of patients who underwent this procedure either for symptomatic lateral compartment knee arthritis or in patients undergoing a joint preservation procedure. This is the first study to our knowledge to look

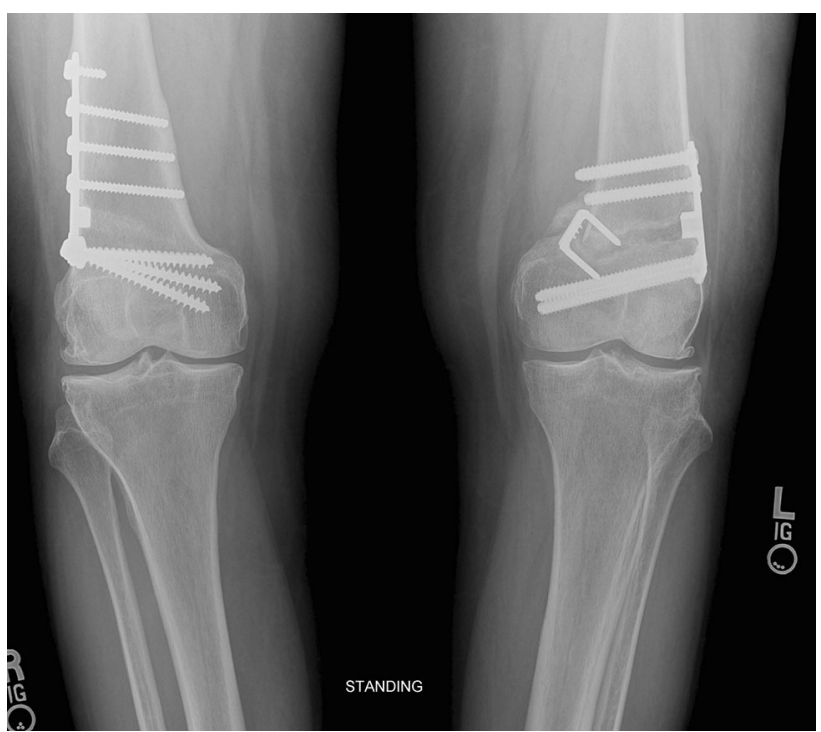

Fig. 3 This AP radiograph shows an osteotomy nonunion (left); note the failure of medial bone hinge.

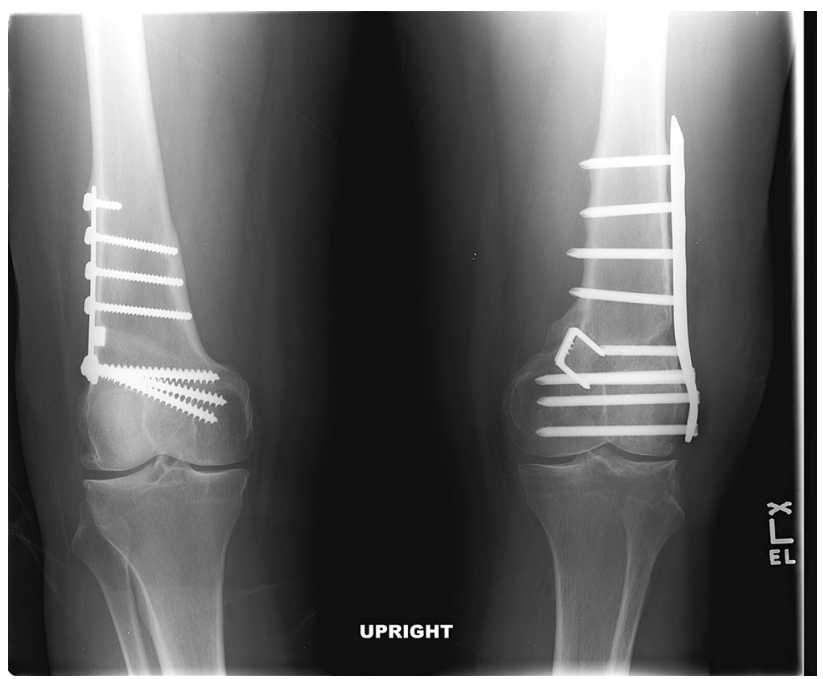

Fig. 4 This AP radiograph demonstrates a healed nonunion (left).

at both of these groups of patients undergoing the same procedure by the same surgeon. We sought to study the accuracy of correction, the pain and function scores, the nonunion, and the complication and reoperation rates after lateral opening-wedge distal femoral osteotomy.

Our study had several limitations. We only report on 21 of 31 knees in regard to alignment correction, because fulllength radiographs were not available on all patients. The small number of patients included in this study makes it difficult to draw conclusions on the data we present. Many of the patients who had postoperative films were not within $3^{\circ}$ of neutral alignment. Further research with larger groups in this area is needed. Second, three different fixation 
Table 4. Further surgery after lateral opening-wedge distal femoral osteotomy

\begin{tabular}{lll}
\hline Further surgery & $\begin{array}{l}\text { Arthritis } \\
\text { group } \\
(\mathrm{N}=19)\end{array}$ & $\begin{array}{l}\text { Joint preservation } \\
\text { group }(\mathrm{N}=12)\end{array}$ \\
\hline Had further surgery & 10 & 6 \\
$\begin{array}{l}\text { Further surgery type* } \\
\text { Manipulation }\end{array}$ & - & 2 \\
Diagnostic arthroscopy & 2 & - \\
Arthroscopy with débridement & 1 & 1 \\
Partial meniscectomy & 1 & - \\
Meniscal repair & - & 1 \\
Hardware removal & 3 & 2 \\
Osteochondral allograft revision & - & 3 \\
Hardware removal, bone & 1 & - \\
grafting, refixation & & 1 \\
Unicompartmental knee & - & - \\
arthroplasty & & \\
Total knee arthroplasty & 5 & \\
\hline
\end{tabular}

* Some knees had more than one further surgery.

devices were used in the series to secure the osteotomy site and insufficient numbers of patients with each device did not allow analysis of a difference in outcome. Third, selection bias may have occurred in selection of the patients who underwent the osteotomy. Joint preservation patients satisfied the criteria for osteotomy as described but were younger patients with a mean age of 26 years and were motivated to maintain an active lifestyle. Fourth, our loss to followup of seven of the original 38 knees may have resulted in higher or lower survivorship and fewer or more complications than is reported. This transfer bias is important to remember when reviewing our results. Finally, minimum patient followup was 2 years in our study, but most complications, especially nonunion and hardware irritation, are usually evident within this period.

We achieved our goal of within $3^{\circ}$ of mechanical neutral alignment in seven of 15 patients in the arthritis group and three of six patients in the joint preservation group who had followup mechanical axis radiographs. This was an unexpected but noteworthy finding. The calculation of $1 \mathrm{~mm}$ of linear correction for $1^{\circ}$ of axial correction may be oversimplified. Intraoperative fluoroscopic and visual analysis of correction to neutral mechanical axis is not as accurate as we had anticipated. It is possible that the limitations of intraoperative fluoroscopy and intraoperative visual analysis of limb alignment in a nonweightbearing situation is that they do not correlate closely enough with preoperative and postoperative weightbearing radiographic alignment measurements. Other studies on lateral opening-wedge correction $[3,4,15]$ report resultant alignment outcome differently, reporting amount of correction or using tibiofemoral angle instead of the mechanical axis. Two studies $[3,4]$ on the lateral opening-wedge technique report a mean improvement in the tibiofemoral angle of $11^{\circ}$ and $6^{\circ}$, respectively. The average correction in mechanical alignment was $5^{\circ}$ valgus and $1^{\circ}$ varus, respectively, Another study on the opening-wedge technique [15] reported that the position of the weightbearing axis through the tibial plateau was changed from $75 \%$ preoperatively to $37 \%$ postoperatively when measured from medial to lateral. Two studies $[1,10]$ on the medial closing-wedge technique report a mean improvement in the tibiofemoral angle of $11^{\circ}$ and $16^{\circ}$, respectively. Based on these studies, a wide variation exists in the amount of correction as well as the final alignment correction achieved. Further studies on alignment correction are needed for clinicians to determine the optimum position of the mechanical axis and to decide whether opening-wedge or closing-wedge osteotomy provides optimal improvement in alignment.

Patients in both groups demonstrated improvements in the IKDC pain and function scores from preoperatively to postoperatively. Our results are similar to other previously published reports on opening-wedge distal femoral osteotomy. Saithna et al. [15] reported on 21 knees that underwent opening-wedge distal femoral osteotomy with followup from 1.6 to 9.2 years. The IKDC score improved from 36 to $53(\mathrm{p}<0.05)$. Dewilde et al. [4] reported on the outcome of opening-wedge distal femoral osteotomy for lateral arthritis of the knee in 19 patients using the Puddu plate and calcium phosphate. Knee Society knee scores improved from 43 to 78 . Das et al. [3] reported on 12 patients with an average age of 52 years undergoing opening-wedge distal femoral osteotomy with the Puddu plate. At 74 months followup, the Lysholm scores improved from 64 to 77 and the clinical Hospital for Special Surgery knee score improved from 42 to 64 . Stahelin et al. [16] reported on 21 medial closing-wedge osteotomies in 19 patients with a mean age of 57 years at 2- to 12-year followup. The authors reported 18 of 19 patients were satisfied. At latest followup, Hospital for Special Surgery knee scores improved from 65 to 84 .

The reoperation rate and survivorship were $53 \%$ and $74 \%$, respectively, for the arthritis group and 50\% and $92 \%$, respectively, for the joint preservation group. There was one nonunion. Saithna et al. [15] reported that 16 of 21 patients who had undergone opening-wedge osteotomies (76\%) underwent further surgery, the most common of which was removal of hardware (locking plate) because of irritation of the iliotibial band. They also reported two cases of loss of correction, one infection, and one nonunion. The 5-year survival with the endpoint of conversion to arthroplasty was $79 \%$. Dewilde et al. [4] reported that four of 19 patients who had an opening-wedge osteotomy underwent hardware removal, one patient underwent 
fracture fixation, and two patients were converted to TKA. Survivorship at 7 years with revision surgery or conversion to TKA as the endpoint was $82 \%$. Das et al. [3] reported one delayed union that prolonged rehabilitation and seven patients who required hardware removal. Survivorship at 74 months with the endpoint of TKA was $83 \%$. Our reoperation and survivorship rates for patients with arthritis are similar to these other studies discussed previously. Importantly, our survivorship in the joint preservation group was higher than any other reported in the literature to date. This may be attributable in part to the younger age of this patient population, but it is an important finding nevertheless. Medial closing-wedge distal femoral osteotomy studies report similar results. Finkelstein et al. [7] reported on 21 knees in 20 patients with a mean 11-year followup. The authors reported a survivorship of $64 \%$ at 10 years, which included seven failures (three early and four late). Sternheim et al. [17] recently reported on the outcome of 45 knees treated with medial closing-wedge distal femoral osteotomy for lateral compartment arthritis. Survivorship at 10,15 , and 20 years was $90 \%, 79 \%$, and $21.5 \%$, respectively. The authors concluded that osteotomy was indicated in younger (mean, 46 years) high-activity patients, but after 20 years most patients were converted to TKA. Wang and Hsu [20] reported on 30 knees undergoing varus osteotomy with a medial blade plate. At an average 99-month followup, $83 \%$ were reported as satisfactory and three were converted to TKA.

Lateral opening-wedge distal femoral osteotomy was less accurate in correction of valgus deformity than we expected, but the procedure was associated with improved pain and function and a 5-year survivorship of $74 \%$ and $92 \%$ in the arthritis and joint preservation patient cohorts, respectively. Predictable healing of the osteotomy was observed. The frequency of hardware removal was higher than we expected and indicates that this should be discussed with patients preoperatively. Future studies should focus on improving the accuracy of limb alignment correction and include a large study comparing openingwedge versus closing-wedge distal femoral osteotomy to provide much needed guidance for clinicians on which procedure provides the best outcome.

\section{References}

1. Aglietti P, Menchetti PP. Distal femoral varus osteotomy in the valgus osteoarthritic knee. Am J Knee Surg. 2000;13:89-95.
2. Backstein D, Morag G, Hanna S, Safir O, Gross A. Long-term follow-up of distal femoral varus osteotomy of the knee. J Arthroplasty. 2007;22:2-6.

3. Das D, Sijbesma T, HJ H, Van Leuven W. Distal femoral opening-wedge osteotomy for lateral compartment osteoarthritis of the knee. Open Access Surg. 2008;1:25-29.

4. Dewilde TR, Dauw J, Vandenneucker H, Bellemans J. Opening wedge distal femoral varus osteotomy using the Puddu plate and calcium phosphate bone cement. Knee Surg Sports Traumatol Arthrosc. 2013;21:249-254.

5. Duivenvoorden T, Brouwer RW, Baan A, Bos PK, Reijman M, Bierma-Zeinstra SM, Verhaar JA. Comparison of closing-wedge and opening-wedge high tibial osteotomy for medial compartment osteoarthritis of the knee: a randomized controlled trial with a six-year follow-up. J Bone Joint Surg Am. 2014;96:1425-1432.

6. Edgerton BC, Mariani EM, Morrey BF. Distal femoral varus osteotomy for painful genu valgum. A five-to-11-year follow-up study. Clin Orthop Relat Res. 1993;288:263-269.

7. Finkelstein JA, Gross AE, Davis A. Varus osteotomy of the distal part of the femur. A survivorship analysis. J Bone Joint Surg Am. 1996;78:1348-1352.

8. Floerkemeier S, Staubli AE, Schroeter S, Goldhahn S, Lobenhoffer P. Outcome after high tibial open-wedge osteotomy: a retrospective evaluation of 533 patients. Knee Surg Sports Traumatol Arthrosc. 2013;21:170-180.

9. Görtz S, Bugbee W. Valgus Malalignment: Diagnosis, Osteotomy Techniques, Clinical Outcomes. Philadelphia, PA, USA: Elsevier Saunders; 2008:896-904.

10. Healy WL, Anglen JO, Wasilewski SA, Krackow KA. Distal femoral varus osteotomy. J Bone Joint Surg Am. 1988;70: 102-109.

11. Jacobi M, Wahl P, Bouaicha S, Jakob RP, Gautier E. Distal femoral varus osteotomy: problems associated with the lateral open-wedge technique. Arch Orthop Trauma Surg. 2011;131:725-728.

12. Kosashvili Y, Safir O, Gross A, Morag G, Lakstein D, Backstein D. Distal femoral varus osteotomy for lateral osteoarthritis of the knee: a minimum ten-year follow-up. Int Orthop. 2010;34: 249-254.

13. Mathews J, Cobb AG, Richardson S, Bentley G. Distal femoral osteotomy for lateral compartment osteoarthritis of the knee. Orthopedics. 1998;21:437-440.

14. Paley D. Principles of Deformity Correction: Berlin, Germany: Springer-Verlag; 2002.

15. Saithna A, Kundra R, Getgood A, Spalding T. Opening wedge distal femoral varus osteotomy for lateral compartment osteoarthritis in the valgus knee. Knee. 2014;21:172-175.

16. Stahelin T, Hardegger F, Ward JC. Supracondylar osteotomy of the femur with use of compression. Osteosynthesis with a malleable implant. J Bone Joint Surg Am. 2000;82:712-722.

17. Sternheim A, Garbedian S, Backstein D. Distal femoral varus osteotomy: unloading the lateral compartment: long-term followup of 45 medial closing wedge osteotomies. Orthopedics. 2011;34:e488-490.

18. Terry GC, Cimino PM. Distal femoral osteotomy for valgus deformity of the knee. Orthopedics. 1992;15:1283-1289; discussion 1289-1290.

19. Thein R, Bronak S, Thein R, Haviv B. Distal femoral osteotomy for valgus arthritic knees. J Orthop Sci. 2012;17:745-749.

20. Wang JW, Hsu CC. Distal femoral varus osteotomy for osteoarthritis of the knee. J Bone Joint Surg Am. 2005;87:127-133. 